\title{
Incidencia de los cebos a base de metaldehído y carbaril en la supervivencia y la reproducción de Folsomia candida
}

Salvio, C., Manetti, P. L., Clemente, N. L. y López, A. N.

\begin{abstract}
RESUMEN
El objetivo del trabajo fue determinar los efectos letales y subletales de metaldehído y/o carbaril sobre Folsomia candida. Se realizaron tres bioensayos bajo un diseño completamente aleatorizado con cuatro repeticiones y en condiciones controladas $\left(20 \pm 2{ }^{\circ} \mathrm{C}\right.$ y $\left.14 \mathrm{~L}: 100\right)$. La unidad experimental fue un recipiente $(9,3 \mathrm{~cm}$ de diámetro y $6,4 \mathrm{~cm}$ de altura) donde se colocaron $60 \mathrm{~g}$ de suelo mezclado con las dosis del cebo y 10 individuos adultos (2 a $3 \mathrm{~mm}$ de longitud). Los tratamientos fueron: 0, 40, 80, 120, 160 y $200 \mathrm{~g}$ de ingrediente activo (IA) ha-1 de metaldehído; 0, 80, 160, 240, 320 y $400 \mathrm{~g} \mathrm{IA}$ ha $^{-1}$ de carbaril; y $0,40+80,80+160,120+240,160+320$ y $200+400 \mathrm{~g} \mathrm{IA} \mathrm{ha}^{-1}$ de metaldehído+carbaril. Con metaldehído tanto la proporción de individuos vivos como el número de crías no difirieron entre los tratamientos a los 7, 14, 21 y 28 días después de la aplicación (DDA) ( $p>0,05)$. Las dosis mayores de carbaril y metaldehído+carbaril ocasionaron disminuciones en la proporción de individuos vivos y en el número de crías a los 28 DDA $(p<0,05)$. Los cebos que contienen carbaril causaron mortalidad y disminución en el número de crías de F. candida.
\end{abstract}

Palabras clave: colémbolos, molusquicida, crustacicida, bioensayos ecotoxicológicos.

Salvio, C.; Manetti, P. L.; Clemente, N. L. and López, A. N., 2018. Effects of metaldehyde and carbaryl based baits on the survival and reproduction of Folsomia candida. Agriscientia 35: 43-50

\section{SUMMARY}

The aim of this work was to determine the lethal and sublethal effects of metaldehyde and/or carbaryl on Folsomia candida. Three bioassays were performed under a completely randomized design with 4 replicates and under controlled conditions $\left(20 \pm 2{ }^{\circ} \mathrm{C}\right.$ y $\left.14 \mathrm{~L}: 100\right)$. The experimental unit consisted of a container $(9.3 \mathrm{~cm}$ diameter and $6.4 \mathrm{~cm}$ high), filled with $60 \mathrm{~g}$ of soil mixed with different doses of bait, in each 10 adult individuals (of 2 to $3 \mathrm{~mm}$ of lenght) were placed. The treatments were: 0; 40; 80; 120; 160 and $200 \mathrm{~g}$ 
active ingredient (a.i.) ha ${ }^{-1}$ of metaldehyde, $0 ; 80 ; 160 ; 240 ; 320$ and $400 \mathrm{~g}$ a.i. ha ${ }^{-1}$ of carbaryl and $0 ; 40+80 ; 80+160 ; 120+240 ; 160+320$ and $200+400$ $\mathrm{g}$ a.i. ha ${ }^{-1}$ metaldehyde+carbaryl. With metaldehyde neither the proportion of live individuals nor the number of offspring differed among treatments at 7, 14, 21 and 28 days after application (DAA) ( $p>0.05)$. The highest doses of carbaryl and metaldehyde+carbaryl showed a decrease in the proportion of live individuals and in the number of offspring at 28 DAA $(p<0.05)$. Baits containing carbaryl caused adult mortality and fewer offspring in F. candida.

Key words: collembolans, molluscicide, crustacicide, ecotoxicological bioassays.

Salvio, C., Manetti, P. L., Clemente, N. L. y López, A. N. Facultad de Ciencias Agrarias, Universidad Nacional de Mar del Plata - Unidad Integrada Balcarce. Ruta 226, km 73,5, Balcarce, Buenos Aires, Argentina, CP: 7620. Correpondencia a: salvio.carla@inta.gob.ar

\section{INTRODUCCIÓN}

Los colémbolos, dentro de los microartrópodos, son unos de los organismos más abundantes en el ecosistema suelo (Hopkin, 1997; Petersen, 2002). Estos organismos son considerados generalistas debido a que su dieta se compone de una mezcla de detritos, algas, bacterias y hongos (Hopkin, 1997; Scheu y Folger, 2004), y debido a su actividad alimentaria, afectan tanto a la microestructura como al proceso de descomposición de los residuos en el suelo (Seastedt, 1984; Cragg y Bardgett, 2001; Fountain y Hopkin, 2005). La modificación de este proceso produce cambios en la mineralización de los nutrientes que en última instancia afectan el crecimiento de las plantas (Moore, Walter y Hunt, 1988; Gange, 2000). Por lo tanto, estos organismos son potenciales bioindicadores que sirven para evaluar el impacto de los plaguicidas en el ambiente edáfico. Dentro de los colémbolos, Folsomia candida (Willem, 1902) (Collembola: Isotomidae) es la especie estandarizada y utilizada en los bioensayos de toxicidad debido a que se puede criar con facilidad, posee una tasa de reproducción elevada y se encuentra presente en todos los tipos de suelo (van Straalen, 1997; van Bruggen y Semenov, 2000; Fountain y Hopkin, 2005; Natal-da-Luz, Römbke y Sousa, 2008).

En la actualidad, la agricultura utiliza una mayor cantidad de plaguicidas para lograr un alto rendimiento en los cultivos y esta situación puede afectar directa o indirectamente a los invertebrados benéficos como los colémbolos (Muthukaruppan, Janardhanan y Vijayalakshmi, 2005; Reinecke y Reinecke, 2007). En consecuencia, cambios en la distribución como en la densidad de estos organismos pueden ser considerados como un indicador de disturbio en el agroecosistema (Carter y Noronha, 2007).

Actualmente, en los sistemas de cultivos bajo siembra directa es frecuente la presencia de babosas (Mollusca: Pulmonata) y bichos bolita (Crustacea: Isopoda) que pueden ocasionar daños a los cultivos de colza, soja y girasol (Clemente, López, Monterubbianesi, Cazzaniga y Manetti, 2008; Garavano et al., 2013). Tanto a nivel mundial como local, el manejo de estos organismos perjudiciales se realiza mediante control químico (Bailey, 2002; Iglesias, Castillejo y Ester, 2002; Mastronardi, 2006; Salvio, Faberi, López, Manetti y Clemente, 2008; Villarino, Manetti, López, Clemente y Faberi, 2011; Garavano et al., 2013), basado principalmente en el uso de cebos tóxicos granulados, formulados con un atrayente alimentario y un ingrediente activo (IA).

Los molusquicidas de mayor uso comercial contienen metaldehído al 4 o $5 \%$ mientras que los crustacicidas, en general contienen carbaril al $8 \%$ y están formulados como cebos granulados (CASAFE, 2015; SENASA, 2017). Por último, existe otro cebo de acción combinada, formulado con metaldehído (4\%) y carbaril (8\%), que permite el control de moluscos y crustáceos (SENASA, 2017). Estos cebos se han desarrollado para ser eficaces a concentraciones bajas y por lo tanto, presentan un efecto adverso mínimo sobre las especies no blanco que comparten el mismo hábitat con los organismos plaga (Cardoso, Santos, Soares y Loureiro, 2015). Sin embargo, existen antecedentes de que los cebos pueden causar efectos letales 
y/o subletales sobre los invertebrados benéficos (Bailey, 2002; Bieri, 2003; Santos, Ferreira, Soares y Loureiro, 2010).

Trabajos previos han demostrado que los cebos formulados con metaldehído no causan efectos letales sobre los enquitreidos, ácaros, colémbolos, sínfilos, carábidos y diplópodos (Büchs, Heimbach y Czarnecki, 1989; Wiltshire y Glen, 1989; Salvio, López, Manetti y Clemente, 2011). Asimismo, metaldehído aplicado a las dosis de registro e incluso superiores, no afecta la supervivencia ni altera el comportamiento de los oligoquetos terrestres (Bieri, Schweizer, Christensen y Daniel, 1989; Bieri, 2003; Iglesias, Castillejo y Castro, 2003; Langan y Shaw, 2006; Edwards, Arancon, Vasko-Bennett, Little y Askar, 2009; Salvio, López, Manetti y Clemente, 2011). Sin embargo, los cebos que poseen carbaril como ingrediente activo resultan tóxicos para los carábidos (Salvio, Manetti, Clemente y López, 2013) y los oligoquetos terrestres (Gupta y Sundararaman, 1991; Mostert, Schoeman y Van der Merwe, 2000; Gupta y Saxena, 2003; Das Gupta, Chakravorty y Kaviraj, 2010; Das Gupta, Chakravorty y Kaviraj, 2011; Lima, Soares y Loureiro, 2011). Teniendo en cuenta los antecedentes antes mencionados, en el presente estudio se evaluaron los efectos letales y/o subletales de tres cebos granulados en base a metaldehído y/o carbaril sobre Folsomia candida.

\section{MATERIALES Y MÉTODOS}

Para los bioensayos se utilizaron ejemplares adultos de Folsomia candida provenientes de una cría masiva. Esta consistió en colocar aproximadamente 30 individuos adultos en recipientes de plástico con tapa, de $9,3 \mathrm{~cm}$ de diámetro y $6,4 \mathrm{~cm}$ de altura, con $3 \mathrm{~cm}$ de suelo húmedo y un soporte de plástico sobre el cual se colocó $1 \mathrm{~g}$ de levadura de cerveza como alimento. Los recipientes se llevaron a una cámara de cría a $20 \pm 2{ }^{\circ} \mathrm{C}$ y un fotoperíodo de 14L: 100 . Semanalmente se revisaron los recipientes para cambiar el alimento y humedecer el sustrato con agua mediante un pulverizador manual.

Las propiedades físicas y químicas del suelo empleado fueron las siguientes: $5,1 \%$ de materia orgánica; $20,9 \%$ de arcilla; 34,9\% de limo; 44,2\% de arena; $\mathrm{pH}=7,7$; conductividad 0,6 mmhos $\mathrm{cm}^{-1}$ y capacidad de intercambio catiónica (CIC) 29,3 meq $100 \mathrm{~g}^{-1}$.

Se realizaron tres bioensayos con los siguientes principios activos formulados como pellets comerciales: metaldehído $4 \%$ (Molusquicida Acay ${ }^{\circledR}$ ), carbaril $8 \%$ (Mata Bibos Acay ${ }^{\circledR}$ ) y metaldehído $4 \%$ + carbaril $8 \%$ (Doble Acay $®$ ) (Acay Agro SRL). Para cada bioensayo se utilizó un diseño completamente aleatorizado con cuatro repeticiones.

En cada bioensayo las unidades experimentales consistieron en recipientes de plástico con tapa, de $9,3 \mathrm{~cm}$ de diámetro y $6,4 \mathrm{~cm}$ de altura. En estos se colocaron $60 \mathrm{~g}$ de suelo húmedo mezclado con las dosis de cada cebo granulado y 10 individuos adultos de F. candida con una longitud corporal de 2 a $3 \mathrm{~mm}$, y posteriormente se mantuvieron en cámara de cría bajo las condiciones mencionadas anteriormente.

Los tratamientos fueron: 0, 40, 80, 120, 160 y 200 g IA de metaldehído ha-1; 0, 80, 160, 240, 320 y 400 g IA de carbaril ha-1 ${ }^{-1}$ y $0,40+80,80+160,120+$ $240,160+320$ y $200+400$ g IA de metaldehído + carbaril ha ${ }^{-1}$. Para lograr la incorporación de cada uno de los cebos granulados se procedió a moler los pellets con un molinillo y se prepararon mezclas para cada dosis. La mezcla sólida consistió en combinar $1 \mathrm{~g}$ de cada uno de los cebos y $99 \mathrm{~g}$ de una sustancia inerte (cebo sin ningún ingrediente activo), de modo tal que se obtuvieron $100 \mathrm{~g}$ de la mezcla y a partir de ella se extrajeron las diferentes dosis. Para la aplicación de cada uno de los cebos se extendió una capa fina y uniforme de suelo de $5 \mathrm{~cm}$ de altura, aproximadamente, sobre un paño de nylon de $50 \mathrm{~cm}$ por $50 \mathrm{~cm}$ de lado. Luego, para lograr una incorporación homogénea del producto al sustrato, se realizaron 20 movimientos de las partes laterales del paño hacia el centro.

Se evaluaron las siguientes variables: el número de individuos vivos y/o muertos a los 7, 14, 21 y 28 días después de la aplicación (DDA) y el número de crías a los 28 DDA. Se realizó el conteo de las crías mediante el método de flotación que consistió en colocar en cada recipiente $200 \mathrm{ml}$ de una mezcla de agua destilada con $0,5 \mathrm{ml}$ de tinta china, que es un colorante no tóxico para los organismos que aumenta el contraste entre el agua y el blanco de los colémbolos (OECD, 2009). Luego se agitó suavemente el suelo con un pincel fino para permitir que los colémbolos puedan flotar hacia la superficie. A continuación, se efectuó la toma de imágenes digitales de los organismos expuestos a cada una de las dosis de los plaguicidas y posteriormente se realizó el conteo.

La proporción de individuos vivos a los 7,14 , 21 y 28 DDA y el número de crías a los 28 DDA se analizaron mediante el análisis de la varianza (ANOVA), previa validación del supuesto de homogeneidad de varianzas mediante la prueba de Levene $(\alpha=0,05)$. Cuando se detectaron diferencias entre las medias se realizó la prueba de 
mínima diferencia significativa o MDS $(\alpha=0,05)$. Los análisis se realizaron con el programa R 3.2.3 (R Development Core Team, 2016).

\section{RESULTADOSY DISCUSIÓN}

En el bioensayo con el cebo que contiene metaldehído no se detectaron diferencias en la proporción de individuos vivos de F. candida entre los tratamientos en ninguna de las fechas de evaluación ( $p>0,05)$ (Figura 1a). La proporción de individuos vivos fue superior a 0,80 en todos los tratamientos. Asimismo, no se encontraron diferencias en el número de crías entre los tratamientos $(p=0,20)$ (Figura $1 b)$. No obstante, en el testigo se hallaron 110 crías y con las dosis mayores de metaldehído (160 y $200 \mathrm{~g}$ de IA) se obtuvieron 73 y 84 crías, respectivamente.

En coincidencia y en condiciones a campo, Salvio et al. (2011) encuentran que la densidad de los colémbolos Onychiuridae e Isotomidae, tanto a los 45 como a los 75 DDA, no es afectada con 160 y
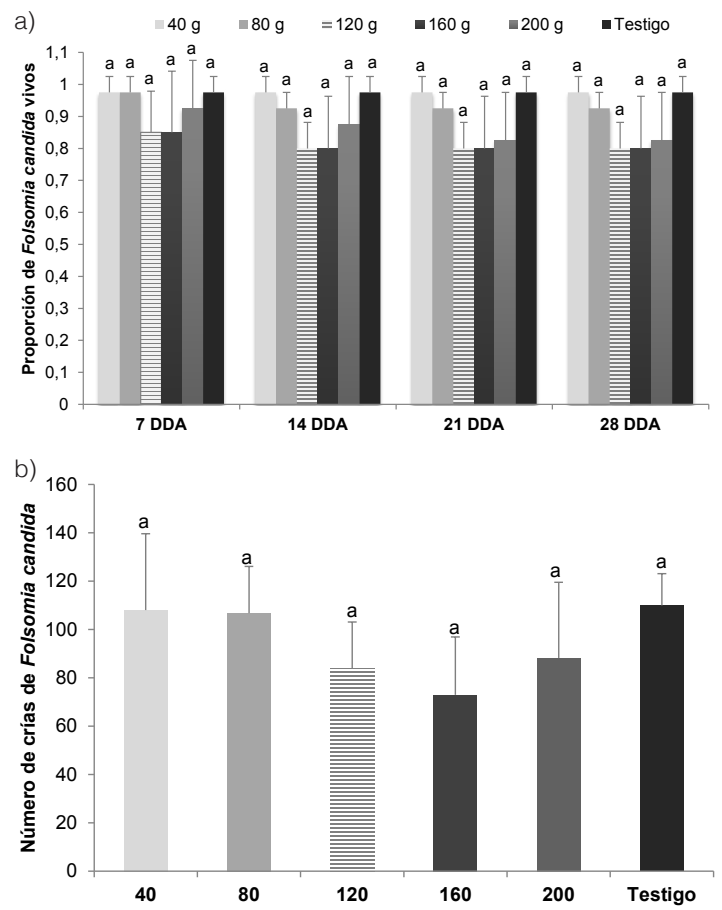

Figura 1. a) Proporción de Folsomia candida vivos (promedio \pm desvío estándar) en los diferentes tratamientos con metaldehído ( $\mathrm{g}$ de IA ha ${ }^{-1}$ ) a los 7, 14, 21 y 28 días después de la aplicación (DDA); y b) Número de crías de $F$. candida en los diferentes tratamientos con metaldehído a los 28 DDA. Barras con letras iguales en cada fecha de observación indican diferencias no significativas entre tratamientos (MDS, $p>0,05$ ).
$200 \mathrm{~g} \mathrm{IA} \mathrm{ha}^{-1}$ de metaldehído. De la misma manera, Salvio et al. (2013) observaron que la aplicación de los cebos en base a metaldehído 4 y $5 \%$ no varía la densidad de los colémbolos detritívoros Onychiuridae y Entomobrydae a los 7, 10, 23 y 37 DDA a las mismas dosis de aplicación. En los sistemas bajo siembra directa, los colémbolos detritívoros, debido a su función, se concentran en la superficie siendo muy sensibles a los efectos adversos ocasionados por los plaguicidas. A pesar de ello, la aplicación de los cebos no afecta a la densidad activa de los detritívoros. Esto es particularmente importante puesto que, este grupo de organismos actúa como catalizador en los procesos de transformación de la materia orgánica (Lavelle, 1997). Además, Iglesias et al. (2003) comprueban que $50 \mathrm{~g}$ de metaldehído ha-1 no disminuye la densidad de estos organismos. Sin embargo, Cardoso et al. (2015) observaron que el cebo en base a metaldehído $5 \%$ causa una disminución significativa en la supervivencia en F. candida a los 28 DDA como así también una reducción en el número de juveniles. No obstante, dichos resultados son detectados con dosis de aplicación superiores a las utilizadas en el presente estudio e incluso a la dosis recomendada a campo. Por lo tanto, hay que tener en cuenta la dosis de aplicación del cebo formulado con metaldehído, ya que con dosis habituales de uso no se afecta la supervivencia y la reproducción de F. candida.

Con respecto al bioensayo con el cebo que contiene carbaril como IA se obtuvieron diferencias en la proporción de individuos vivos entre los tratamientos a los 21 y 28 DDA, $p=0,0045$ y $p=$ 0,0019 , respectivamente (Figura 2a). A los 21 DDA, se observó con la dosis mayor (400 g de carbaril) una disminución en la proporción de individuos vivos, diferenciándose de los demás tratamientos $(p<0,05)$ (Figura 2a). Por otro lado, a los 28 DDA la proporción de individuos vivos fue mayor en el testigo y con la dosis menor con un valor de 0,975, mientras que, con la dosis mayor se produjo una disminución significativa con un valor de 0,375 (Figura 2a)

Con respecto al número de crías se detectaron diferencias significativas entre los tratamientos a los 28 DDA ( $p=0,0098)$. Como se observa en la Figura $2 b$, el número mayor de crías se obtuvo en el testigo y con las dosis menores 80 y $160 \mathrm{~g}$ de carbaril con un promedio de 110 crías; en cambio, con la dosis mayor $400 \mathrm{~g}$ de carbaril se obtuvo un número menor, 54 crías.

A diferencia de las observaciones efectuadas en el presente estudio, Salvio et al. (2011) en condiciones a campo encuentran que la densidad de los colémbolos tanto a los 45 como a los 75 

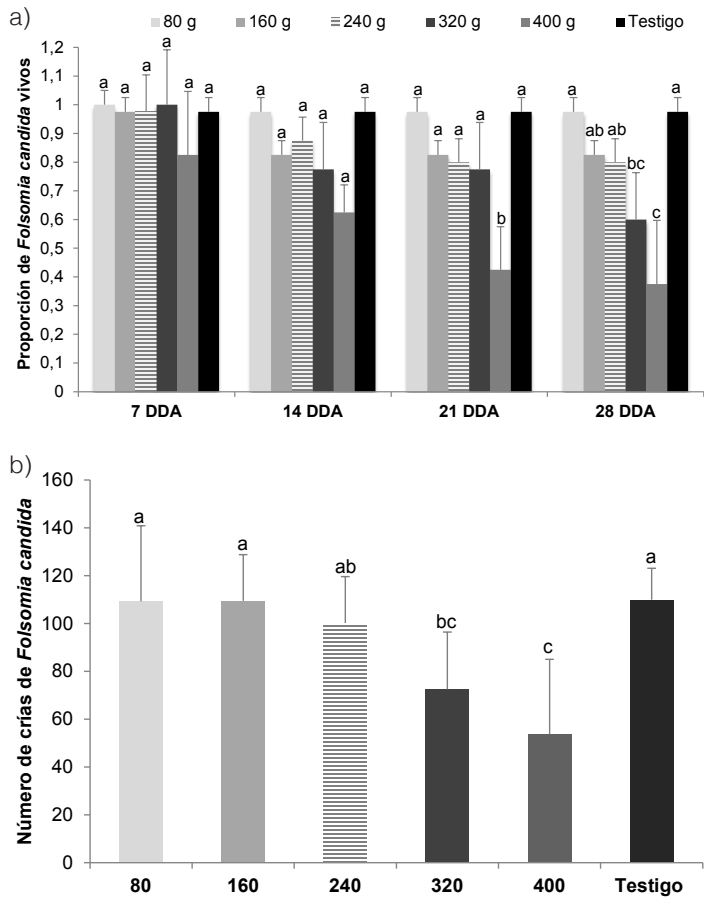

Figura 2. a) Proporción de Folsomia candida vivos (promedio \pm desvío estándar) en los diferentes tratamientos con carbaril ( $\mathrm{g}$ de $\mathrm{IA} \mathrm{ha}^{-1}$ ) a los 7, 14, 21 y 28 días después de la aplicación (DDA); y b) Número de crías de $F$. candida en los diferentes tratamientos con carbaril a los 28 DDA. Barras con letras iguales en cada fecha de observación indican diferencias no significativas entre tratamientos (MDS, $\mathrm{p}>0,05$ ).

DDA, no varía con $320 \mathrm{~g}$ de carbaril de dos cebos comerciales formulados a un $8 \%$. De manera similar, la densidad de los colémbolos detritívoros Onychiuridae y Entomobrydae no cambió con los cebos en base a carbaril a las mismas dosis de aplicación a los 7, 10, 23 y 37 DDA (Salvio et al., 2013). Por otra parte, considerando el carbamato metiocarb formulado como cebo al $4 \%$, Cardoso et al. (2015) observaron una disminución significativa en el número de juveniles de $F$. candida. Sin embargo, no ocasiona efectos letales en el colémbolo con dosis comprendidas entre 5 y $54 \mathrm{~kg}$ de IA ha-1, es decir dosis superiores a las utilizadas habitualmente.

Cabe destacar que cuando el carbaril es aplicado como insecticida causa efectos letales sobre los colémbolos (Thompson y Gore, 1972; Joy y Chakravorty, 1991). En efecto, Spain (1974) y Schulze et al. (2001) encontraron que el carbaril disminuye la densidad de los colémbolos. En coincidencia, los cebos que contienen carbaril como IA, ante las dosis mayores se comportaron de manera similar a la aplicación del insecticida, debido a que ocasionaron mortalidad y disminución del número de crías sobre F. candida (Figura 2).

En el bioensayo con el cebo de acción doble metaldehído y carbaril, se obtuvieron diferencias en la proporción de colémbolos vivos entre los tratamientos a los $7,14,21$ y 28 DDA $(p<0,05)$. En todas las observaciones, la proporción mayor de individuos vivos se obtuvo en el testigo y con las dosis menores del cebo (Figura 3a), mientras que las proporciones menores se alcanzaron con las dosis mayores (Figura 3a). Además, en estos tratamientos la proporción disminuyó a medida que aumentó el número de días después de la aplicación.

Por otra parte, el número de crías difirió entre los tratamientos a los 28 DDA ( $p<0,0001)$, hallándose el número mayor en el testigo y el menor con $200+$ $400 \mathrm{~g}$ de metaldehído + carbaril, siendo sus valores 110 y 48 crías, respectivamente (Figura 3b).

A diferencia de las observaciones efectuadas en el presente estudio, Salvio et al. (2011) encuentran
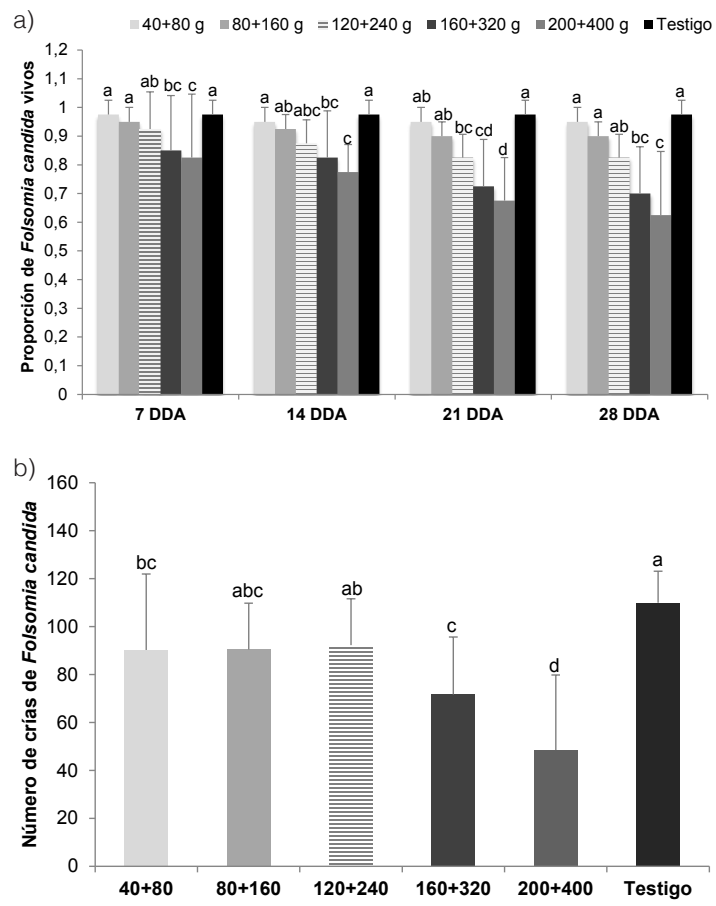

Figura 3. a) Proporción de Folsomia candida vivos (promedio \pm desvío estándar) en los diferentes tratamientos con metaldehído + carbaril (g de IA ha-1) a los 7, 14, 21 y 28 días después de la aplicación (DDA); y b) Número de crías de F. candida en los diferentes tratamientos con metaldehído + carbaril a los 28 DDA. Barras con letras iguales en cada fecha de observación indican diferencias no significativas entre tratamientos (MDS, p> 0,05). 
que la densidad de los colémbolos y su composición taxonómica, tanto a los 45 como a los 75 DDA, no es afectada con $240+480 \mathrm{~g}$ metaldehído + carbaril. Asimismo, en condiciones a campo, Salvio et al. (2013) comprobaron que metaldehído + carbaril no varía el número de los colémbolos detritívoros Onychiuridae y Entomobrydae a los 7, 10, 23 y 37 DDA. Si bien los cebos son efectivos sobre los organismos plaga (es decir, las babosas y los bichos bolita) hay que tener en cuenta que aquellos que contienen a carbaril como IA en su formulación causaron efectos letales como subletales sobre los colémbolos (Figuras 2 y 3 ).

Por otra parte, se encuentran diversos resultados en estudios con los cebos granulados sobre otros invertebrados benéficos. Considerando a los oligoquetos terrestres, se detectó que metaldehído aplicado en forma de pellets a las dosis utilizadas en el campo, e incluso superiores, no afecta la supervivencia, ganancia de peso ni el comportamiento de estos organismos (Bieri et al., 1989; Bieri, 2003; Iglesias et al., 2003; Langan y Shaw, 2006; Edwards et al., 2009; Salvio et al., 2011). Resultados similares se observaron con los cebos en base a metaldehído y/o carbaril (Salvio, 2012).

Ahora bien, cabe destacar que los cebos en base a carbaril en condiciones a campo causaron una disminución significativa en la densidad de Scarites anthracinus, carábido predador de estados juveniles de babosas y bichos bolita (Salvio et al., 2013). Asimismo, se ha determinado que aquellos cebos ocasionan efectos letales sobre el carábido. Es decir, para esta especie la dosis letal cincuenta $\left(\mathrm{DL}_{50}\right)$ del formulado que contiene carbaril es de $2,95 \mathrm{~kg} \mathrm{ha}^{-1}$, equivalente a la dosis que habitualmente se aplica en el campo (Salvio, 2012). Con respecto al cebo combinado, la $D L_{50}$ es de $1,54 \mathrm{~kg}$ de metaldehído + carbaril ha-1, la mitad de la dosis de aplicación recomendada (Salvio, 2012). En coincidencia con estos resultados, en este estudio se observó que los cebos formulados con carbaril afectan a los colémbolos (Figuras 2 y 3) y por lo tanto, estos deberían utilizarse con precaución.

\section{CONCLUSIÓN}

Los cebos que contienen carbaril como IA causaron mortalidad sobre F. candida como así también disminución en el número de crías. Teniendo en cuenta que estos formulados ocasionan efectos adversos sobre el colémbolo, se debería ajustar adecuadamente la dosis de aplicación en función a la densidad de las plagas. De esta manera, se intentaría disminuir los disturbios en el equilibrio biológico de los organismos que viven en el sistema suelo.

\section{BIBLIOGRAFÍA}

Bailey, S. (2002). Molluscicidal baits for control of terrestrial gastropods. En G. M. Barker (Ed.), Molluscs as crop pests (33-54). Hamilton, New Zealand: CABI Publishing.

Bieri, M., Schweizer, H., Christensen, K. y Daniel, O. (1989). The effect of metaldehyde and methiocarb slug pellets on Lumbricus terrestris $L$. En I. Henderson (Ed.), Slugs and snails in world agriculture (237-244). Thornton Heath, Londres, Inglaterra: British Crop Protection Council.

Bieri, M. (2003). The environmental profile of metaldehyde. En BCPC Symposium Proceedings (255-262), Thornton Heath, Londres, Inglaterra: British Crop Protection Council.

Büchs, W., Heimbach, U. y Czarnecki, E. (1989). Effects of snail baits on non-target carabid beetles. Monograph. British Crop Protection Council, 41, 245-252.

Cardoso, D. N., Santos, M. J. G., Soares, A. M. V. M. y Loureiro, S. (2015). Molluscicide baits impair the life traits of Folsomia candida (Collembola): Possible hazard to the population level and soil function. Chemosphere, 132, 1-7.

Carter, M. R. y Noronha, C. (2007). Soil microarthropod communities and microbial parameters in the potato ridge under two field management systems on sandy loams in Atlantic Canada. Canadian Journal of Soil Science, 87 (4), 399-404.

CASAFE (2015). Cámara de Sanidad Agropecuaria y fertilizantes. Recuperado de http:// www.casafe.org.

Clemente, N. L., López, A. N., Monterubbianesi, M. G., Cazzaniga, N. J. y Manetti, P. L. (2008). Biological studies and phenology of slug Deroceras reticulatum (Müller, 1774) (Pulmonata: Stylommatophora). Invertebrate Reproduction and Development, 52, 23-30.

Cragg, R. y Bardgett, R. D. (2001). How changes in soil faunal diversity and composition within a trophic group influence decomposition processes. Soil Biology and Biochemistry, 33, 2073-2081.

Das Gupta, R., Chakravorty, P. P. y Kaviraj, A. (2010). Studies on relative toxicities of six insecticides on epigeic earthworm, Perionyx excavatus. Bulletin of Environmental Contamination and Toxicology, 85, 83-86.

Das Gupta, R., Chakravorty, P. P. y Kaviraj, A. 
(2011). Susceptibility of epigeic earthworm Eisenia fetida to agricultural application of six insecticides. Chemosphere, 84 (5), 724-726.

Edwards, C. A., Arancon, N. Q., Vasko-Bennett, M., Little, B. y Askar, A. (2009). The relative toxicity of metaldehyde and iron phosphate-based molluscicides to earthworms. Crop Protection, 28 (4), 289-294.

Gange, A. (2000). Arbuscular mycorrhizal fungi, Collembola and plant growth. Trends in Ecology and Evolution, 15, 369-372.

Garavano, M. E., Manetti, P. L., Clemente, N. L., Faberi, A. J., Salvio, C. y López, A. N. (2013). Cebos molusquicidas y molusquicidas líquidos para el control de Deroceras reticulatum (Pulmonata: Stylomatophora), plaga en el cultivo de colza. Revista de Investigaciones Agropecuarias del Instituto Nacional de Tecnología Agropecuaria, 39 (1), 60-66.

Fountain, M. T. F. y Hopkin, S. P. (2005). Folsomia candida (Collembola): A "Standard" Soil Arthropod. Annual Review of Entomology, 50, 201-22.

Gupta, S. K. y Saxena, P. N. (2003). Carbarylinduced behavioural and reproductive abnormalities in the earthworm Metaphire posthuma: a sensitive model. Alternatives to Laboratory Animals, 31 (6), 587-593.

Gupta, S. K. y Sundararaman, V. (1991). Correlation between burrowing capability and AchE activity in the earthworm Pheretima posthuma, on exposure to carbaryl. Bulletin of Environmental Contamination and Toxicology, 46, 859-865.

Hopkin, S. P. (1997). Biology of the Springtails (Insecta, Collembola). Oxford, Inglaterra: Oxford University Press,

Iglesias, J., Castillejo, J. y Ester, A. (2002). Laboratory evaluation of potencial molluscicides for he control of eggs of the pest slug Deroceras reticulatum (Müller) (Pulmonata: Limacidae). International Journal of Pest Management, 48 (1), 19-23.

Iglesias, J., Castillejo, J. y Castro, R. (2003). The effects of repeated applications of the molluscicide metaldehyde and the biocontrol nematode Phasmarhabditis hermaphrodita on molluscs, earthworms, nematodes, acarids and collembolans: a two-year study in north-west Spain. Pest Management Science, 59 (11), 1217-1224.

Joy, V. C. y Chakravorty, P. P. (1991). Impact of insecticicides on nontarget microasthropod fauna in agricultural soil. Ecotoxicology and Environmental Safety, 22, 8-16.

Langan, A. M. y Shaw, E. M. (2006). Responses of the earthworm Lumbricus terrestris (L.) to iron phosphate and metaldehyde slug pellet formulations. Applied Soil Ecology, 34 (2-3), 184189.

Lavelle, P. (1997). Faunal activities and soil processes: adaptive strategies that determine ecosystem function. Advances in Ecological Research, 27, 93-132.

Lima, M. P. R., Soares, A. M. V. M. y Loureiro, S. (2011). Combined effects of soil moisture and carbaryl to earthworms and plants: Simulation of flood and drought scenarios. Environmental Pollution, 159, 1844-1851.

Mastronardi, F. (2006). Control químico de isópodos y babosas en un cultivo de girasol bajo siembra directa (Tesis de grado). Facultad de Ciencias Agrarias. Universidad Nacional de Mar del Plata. Balcarce, Argentina.

Moore, J. C., Walter, D. E. y Hunt, H. W. (1988). Arthropod regulation of micro-and mesobiota in below-ground detrital food webs. Annual Review of Entomology, 33, 419-439.

Mostert, M. A., Schoeman, A. S. y Van der Merwe, M. (2000). The toxicity of five insecticides to earthworms of the Pheretima group, using an artificial soil test. Pest Management Science, 56, 1093-1097.

Muthukaruppan, G., Janardhanan, S. y Vijayalakshmi, G. S. (2005). Sublethal toxicity of the herbicide butachlor on the earthworm Perionyx sansibaricus and its histological changes. Journal of Soils and Sediments, 5 (2), 82-86.

Natal-da-Luz, T., Römbke, J. y Sousa, J. P. (2008). Avoidance tests in site specific risk assessment: influence of soil properties on the avoidance response of collembolan and earthworms. Environmental Toxicology and Chemistry, 27, 11121117.

Petersen, H. (2002). General aspects of collembolan ecology at the turn of the millennium. Pedobiologia, 46, 246-260.

R Development Core Team. (2016). R: a language and environment for statistical computing. R Foundation for Statistical Computing, Vienna, Austria. Version R 3.2.3.

Reinecke, S. y Reinecke, A. (2007). Biomarker response and biomass change of earthworms exposed to chlorpyrifos in microcosms. Ecotoxicology and Environmental Safety, 66, 92101.

Salvio, C., Faberi, A. J., López, A. N., Manetti, P. L. y Clemente, N. L. (2008). The efficacy of three metaldehyde pellets marketed in Argentina, 
on the control of Deroceras reticulatum (Müller) (Pulmonata: Stylommatophora). Spanish Journal of Agricultural Research, 6 (1), 70-77.

Salvio, C., López, A. N., Manetti, P. L. y Clemente, N. L. (2011). Effects of granulated baits on meso and macrofauna in soybean soil system. Journal of Environmental Biology, 32 (6), 793-799.

Salvio, C. (2012). Impacto de las prácticas agrícolas sobre poblaciones de Milax gagates y Armadillidium vulgare e invertebrados benéficos en soja bajo siembra directa (Tesis de maestría). Faculta de Ciencias Agrarias. Universidad Nacional de Mar del Plata, Argentina.

Salvio, C., Manetti, P. L., Clemente, N. L. y López, A. N. (2013). Acción de los cebos granulados sobre los invertebrados edáficos. Ciencia del Suelo, 31 (2), 165-174.

Santos, M., Ferreira, N. C., Soares, A. V. M. y Loureiro, S. (2010). Toxic effects of molluscicidal baits to the terrestrial isopod Porcellionides pruinosus (Brandt, 1833). Journal of Soils and Sediments, 10 (7), 1335-1343.

Scheu, S. y Folger, M. (2004). Single and mixed diets in Collembola: effects on reproduction and stable isotope fractionation. Functional Ecology, 18, 94-102.

Schulze, T. L., Jordan, R. A., Hung, R. W., Krivenko, A. J., Schulze, J. J. y Jordan, T. M. (2001). Effects of an application of granular carbaryl on nontarget forest floor arthropods. Journal of Economic Entomology, 94, 123-128.

Seastedt, T.R. (1984). The role of microarthropods in decomposition and mineralization processes. Annual Review of Entomology, 29, 25-46.

SENASA (2017). Servicio Nacional de Sanidad y Calidad Agroalimentaria. Recuperado de http:// www.senasa.gob.ar/informacion/prod-vet-fito-yfertilizantes/prod-fitosanitarios-y-fertili/registronacional-de-terapeutica-vegetal

Spain, A. V. (1974). The effects of carbaryl and DDT on the litter fauna of a Corsican pine forest (Pinus nigra var. maritima): A multivariate comparison. Journal of Applied Ecology, 11, 467481.

Thompson, A. R. y Gore, F. L. (1972). Toxicity of twenty-nine insecticides to Folsomia candida: Laboratory Studies. Journal of Economic Entomology, 65, 1255-1260.

Van Bruggen, A. H. C. y Semenov, A. M. (2000). In search of biological indicators for soil health and disease suppression. Applied Soil Ecology, 15 (1), 13-24.

Van Straalen, N. M. (1997). Community structure of soil arthropods, bioindicator of soil quality. En C. E., Pankhurst, B. M., Doube, y V. V. S. R., Gupta (Eds.), Bioindicators of Soil Health (235-264). Wallingford, Connectituc, Estados Unidos: CAB International.

Villarino, S. V., Manetti, P. L., López, A. N., Clemente, N. L. y Faberi, A. J. (2011). Formulaciones con combinación de ingredientes activos para el control de Armadillidium vulgare (Crustacea: Isopoda), plaga en el cultivo de colza. Revista de Investigaciones Agropecuarias del Instituto Nacional de Tecnología Agropecuaria, 30 (1), 91-96.

Wiltshire, C. W. y Glen, D. M. (1989). Effects of molluscicides on slugs and soil arthropods in winter cereal crops. Monograph. British Crop Protection Council, 41, (pp. 399-406). 